\title{
A compound heterozygous mutation in HADHB gene causes an axonal Charcot-Marie-tooth disease
}

Young Bin Hong ${ }^{1}$, Ja Hyun Lee ${ }^{2}$, Jin-Mo Park ${ }^{3}$, Yu-Ri Choi ${ }^{4}$, Young Se Hyun ${ }^{2}$, Bo Ram Yoon ${ }^{2}$, Jeong Hyun Yoo ${ }^{5}$, Heasoo Koo ${ }^{6}$, Sung-Chul Jung ${ }^{4}$, Ki Wha Chung ${ }^{2^{*}}$ and Byung-OK Choi ${ }^{1^{*}}$

\begin{abstract}
Background: Charcot-Marie-Tooth disease (CMT) is a heterogeneous disorder of the peripheral nervous system. So far, mutations in hydroxyacyl-CoA dehydrogenase/3-ketoacyl-CoA thiolase/enoyl-CoA hydratase (trifunctional protein), beta subunit $(H A D H B)$ gene exhibit three distinctive phenotypes: severe neonatal presentation with cardiomyopathy, hepatic form with recurrent hypoketotic hypoglycemia, and later-onset axonal sensory neuropathy with episodic myoglobinuria.

Methods: To identify the causative and characterize clinical features of a Korean family with motor and sensory neuropathies, whole exome study (WES), histopathologic study of distal sural nerve, and lower limb MRIs were performed.

Results: WES revealed that a compound heterozygous mutation in HADHB is the causative of the present patients. The patients exhibited an early-onset axonal sensorimotor neuropathy without episodic myoglobinuria, and showed typical clinical and electrophysiological features of CMT including predominant distal muscle weakness and atrophy. Histopathologic findings of sural nerve were compatible with an axonal CMT neuropathy. Furthermore, they didn't exhibit any other symptoms of the previously reported HADHB patients.

Conclusions: These data implicate that mutation in HADHB gene can also cause early-onset axonal CMT instead of typical manifestations in mitochondrial trifunctional protein (MTP) deficiency. Therefore, this study is the first report of a new subtype of autosomal recessive axonal CMT by a compound heterozygous mutation in $H A D H B$, and will expand the clinical and genetic spectrum of HADHB.
\end{abstract}

Keywords: Charcot-Marie-Tooth disease (CMT), Whole exome sequencing (WES), HADHB, Mitochondrial trifunctional protein (MTP)

\section{Background}

Charcot-Marie-Tooth disease (CMT), also called hereditary motor and sensory neuropathy, is a clinically and genetically heterogeneous disorder of the peripheral nervous system [1-3]. CMT is conventionally divided into the demyelinating form (CMT1) and the axonal form (CMT2). Of them, CMT2 has been divided into many

\footnotetext{
* Correspondence: kwchung@kongju.ac.kr; bochoi@skku.edu

${ }^{2}$ Department of Biological Science, Kongju National University, 182 Sinkwan-dong, Gongju 314-701, Korea

'Department of Neurology, Samsung Medical Center, Sungkyunkwan University School of Medicine, 50 Ilwon-dong Gangnam-Gu, Seoul 135-710, Korea

Full list of author information is available at the end of the article
}

subtypes (from CMT2A to CMT2P) according to their distinct clinical symptoms and genetic causes [4-6].

So far, more than 60 causative genes or loci have been reported to be associated with the CMT development (http://www.molgen.ua.ac.be/CMTMutations/Home/ Default.cfm). However, large number of CMT patients is waiting for uncovering their specific genetic underlying causes. Thus efficient analysis tools such as whole exome sequencing (WES) are required to accelerate the identification of the underlying genetic causes. Applications of WES to CMT have been proved as to be powerful to identify rare genetic causes from small-sized pedigrees [7-9].

\section{Biomed Central}

(c) 2013 Hong et al.; licensee BioMed Central Ltd. This is an open access article distributed under the terms of the Creative Commons Attribution License (http://creativecommons.org/licenses/by/2.0), which permits unrestricted use, distribution, and reproduction in any medium, provided the original work is properly cited. 
Mitochondrial trifunctional protein (MTP), a constituent of inner membrane of mitochondria, exerts a significant catalytic activity for beta-oxidation cycle, which metabolizes long-chain fatty acyl-CoA [10]. MTP consists of heterooctamer $(\alpha 4 \beta 4)$ possessing three different enzyme activities: long-chain enoyl-CoA hydrolase (LCEH) and long-chain 3-hydroxy-acyl-CoA dehydrogenase (LCHAD) activities harbored by the $\alpha$-subunit (HADHA), while $\beta$-subunit (HADHB) encode longchain 3-ketoacyl-CoA thiolase (LCKT) [10]. Deficiency of MTP is an autosomal recessive disorder that exhibits characteristic features of cardiomyopathy, hypoketotic hypoglycemia, metabolic acidosis, sudden infant death, metabolic encephalopathy, liver dysfunction, axonal sensory neuropathy and pigmentary retinopathy [11-13].

Here, we report clinical and pathological features of an autosomal recessive CMT family harboring a novel compound heterozygous $H A D H B$ mutation, which is identified by WES.

\section{Methods \\ Patients}

We enrolled a total of 6 members of a Korean demyelinating CMT family (family ID: FC354) with 2 affected individuals. The CMT phenotype of this family was seemed to be inherited with autosomal recessive mode, since two of three siblings were affected while both parents were unaffected. No CMT patient was identified from close relatives of the family. This study also recruited 500 Korean healthy controls with no familial history of neuromuscular disorders (10-60 years old). All samples were collected from 2011 to 2012. Informed consent was obtained from all participants and from parents of participants younger than 18 years of age according to the protocol approved by the Institutional Review Board for Ewha Womans University, Mokdong Hospital (ECT 11-58-37).

\section{Clinical and electrophysiological assessments}

Patients were evaluated by taking a detailed history including motor and sensory impairments, deep tendon reflexes, and muscle atrophy undertaken by two independent neurologists. Muscle strengths of flexor and extensor muscles were assessed manually using the medical research council (MRC) scale. In order to determine physical disability, we used three scales, a functional disability scale (FDS) [14], and a CMT neuropathy score (CMTNS) [15]. Sensory impairments were assessed in terms of the level and severity of pain, temperature, vibration and position. Nerve conduction studies (NCSs) were carried out with a surface electrode in median, ulnar, peroneal, tibial, and sural nerves as previously described [16].

\section{MRI studies}

Two patients (II-1 and -2) with $H A D H B$ mutation were studied with MRI of the brain, hip, thigh and lower leg using a 1.5-T system (Siemens Vision, Siemens, Germany). Whole brains were scanned using a slice thickness of $7 \mathrm{~mm}$ and a 2-mm interslice gap, to produce 16 axial images. The imaging protocol consisted of T2-weighted spin echo $(\mathrm{TR} / \mathrm{TE}=4,700 / 120 \mathrm{~ms})$, T1-weighted spin echo (TR/TE $=550 / 12 \mathrm{~ms})$, and fluid-attenuated inversion recovery (FLAIR) $(\mathrm{TR} / \mathrm{TE}=9,000 / 119 \mathrm{~ms}$, inversion time 2,609 $\mathrm{ms}$ ) images. Lower leg imaging was carried out in axial [field of view (FOV) 24-32 cm, slice thickness $10 \mathrm{~mm}$, and slice gap $0.5-1.0 \mathrm{~mm}$ ] and coronal planes (FOV $38-40 \mathrm{~cm}$, slice thickness 4-5 mm, slice gap 0.5-1.0 mm).

\section{Histopathological studies}

Histopathological analysis of the distal sural nerve was performed in a patient (II-2). The density of myelinated fibers (MFs), axonal diameter, and myelin thickness were determined directly from the semi-thin transverse sections using a computer-assisted image analyzer (AnalySIS, Soft Imaging System, Germany). Ultrathin cut samples (60 $65 \mathrm{~nm}$ ) are contrasted with uranyl acetate and lead citrate for ultrastructural study (H-7650, Hitachi, Japan).

\section{DNA preparation and whole exome sequencing}

Total DNA was purified from peripheral blood using QIAamp blood DNA purification kit (QIAGEN, Hilden, Germany). DNAs were prescreened for duplication of 17p12 (PMP22) and mutations in the coding exons of GJB1, MPZ, NEFL and MFN2 as previously described [9]. Exome sequencing and subsequent filtering was performed as previously described [9].

\section{Determination of exon5 splicing and DNA cloning}

Total mRNA was purified from proband's fibroblast using RNeasy minikit (QIAGEN). Then cDNA was synthesized using Superscript reverse transcriptase (Invitrogen, Carlsbad, CA). For amplification of wildtype and mutant HADHB, following primers are used: HADHB forward, 5' -ACG TCA GCC AAG ATT CCA GA-3', and HADHB reverse, 5'-GCA CAG AAA CTT CAG GTC ACT TC-3'. Sequencing was performed to determine cDNA sequence of each amplified mutant allele. Amplified cDNAs were cloned into pCR2.1-TOPO vector (Invitrogen) and subsequently moved into expression vector, pCMV-myc (Clontech, Mountain View, CA).

\section{DNA transfection and determination of protein half-life}

HEK293 cells $\left(2 \times 10^{5}\right)$ were transfected with control vector, pCMV-myc, and cloned HADHB cDNAs using FuGene transfection reagent (Promega, Madison, WI). For determination of half-life of HADHB, cyclohexamide $(100 \mu \mathrm{M})$ was treated after $20 \mathrm{hr}$ of transfection. 


\section{Western blotting}

Protein expression in fibroblast and HEK293 cells were determined by standard Western blotting. Anti-HADHB Ab (Santa Cruz Biotechnology, Santa Cruz, CA), anti-myc Ab (Abcam, Cambridge, UK), anti-actin Ab, anti-mouse secondary Ab, anti-rabbit secondary Ab (Sigma, St. Louis, MO), and ECL plus Western blotting substrate (Thermo Scientific, Rockford, IL) were used for detection of protein.

\section{Results}

Identification of a compound heterozygous mutation in HADHB

To identify the underlying genetic cause of this family, WES was performed for five members (I-1, I-2, II-1, II-2, and II-4). Total sequencing yields was $10.11 \mathrm{Gbp} /$ sample, and mappable reads were $93.1 \%$. Total number of variants (SNPs and indels) was 80,301 single nucleotide variants (SNVs)/sample, of which 20,430 SNVs were coding variants (Additional file 1: Table S1). Several functionally significant variants were identified in the CMT-relevant genes; however, no variant was fitted for autosomal recessive inheritance of the family (Additional file 1: Table S2). Moreover, most of them were also observed in controls.

From the unreported or rare functionally significant SNVs reported in dbSNP135 and 1000 Genome project database, we could identify two pairs of compound heterozygous mutations transmitted from each of the parents: $[\mathrm{c} .210-1 \mathrm{G}>\mathrm{C}]+[\mathrm{c} .686 \mathrm{G}>\mathrm{T}]$ in HADHB (NM_000183)

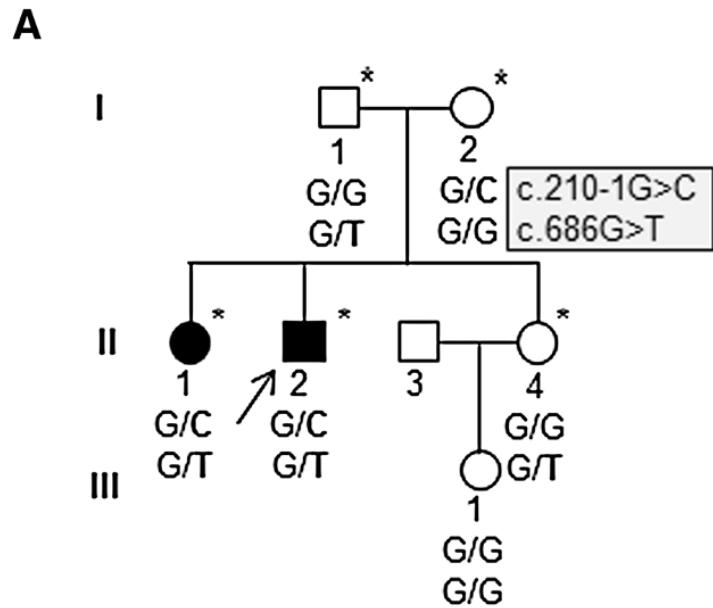

B

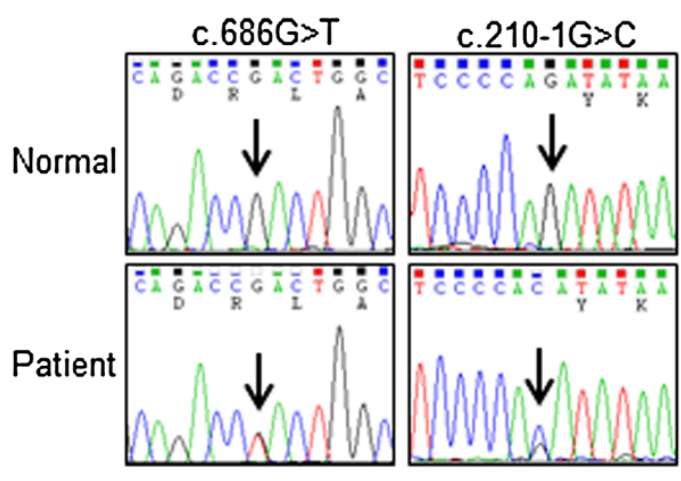

D
C
H. sapiens (NP_000174.1)
SETM GHS ADRLA A A F A V SR L
M. musculus (NP_663533.1) NETM GH S ADRLA A A F A V SRM
R. norvegicus (NP_598302.1) NETM GH S AD RLA A A F A V SRM
B. taurus (NP_776761.1)
X. tropicalis (NP_989142.1)
X. laevis (NP_001080077.1)
D. rerio (NP_956313.1)
SETM GHS ADRLA A A F A ISRE
SETM GHS ADRLA A A FS V SR V
SESM GHSADRLAAAFS VSR V
AETM GHS ADRLA A A FG VSR L

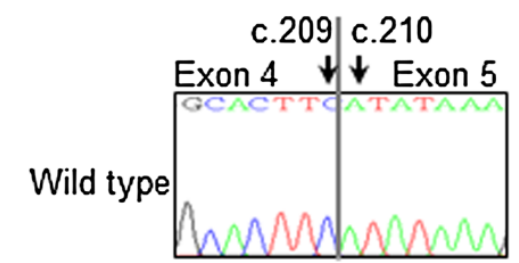

$\mathbf{E}$

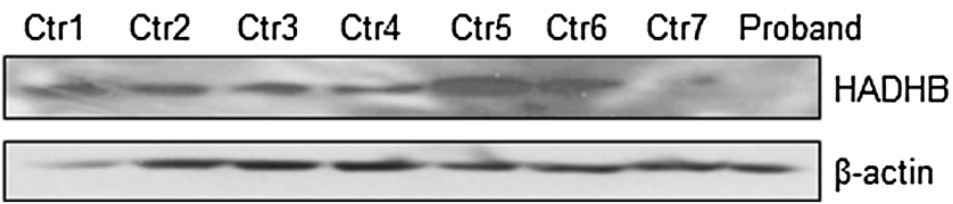

Figure 1 Pedigree and HADHB mutations in FC354. (A) Alleles of two HADHB mutations were indicated. Open symbols, unaffected; filled symbols, affected; arrow, proband; asterisks, individuals underwent WES. (B) Sequencing chromatograms of c.686G > T and c.210-1G > C mutation. Vertical arrows indicate the mutation site. (C) Conservation analysis of amino acid sequences among species. Complete conserved amino acids, red; partial conserved amino acids, blue. (D) Deletion of exon5 in c.210-1G > C allele was confirmed by cDNA sequencing. (E) Expression level of HADHB in the proband's fibroblast. Ctr1, normal control fibroblast; and Ctr2-7, fibroblast from CMT patients with mutation in other than HADHB gene. 
and [c.C935T] + [c.697_698insTT] in Cyclin-dependent kinase-like 4 (CDKL4, NM_001009565). Since the CDKL4 compound mutations were found in Korean healthy controls, the HADHB compound heterozygous mutation was finally considered as the underlying cause of the axonal CMT (Figures 1A and B). The p.Arg229Leu mutation site locating in the thiolase $\mathrm{N}$ domain is highly conserved between different species (Figure 1C) and is predicted to affect the functional integrity by in silico analysis (SIFT and PolyPhen2). The other mutation, c.210-1G > C, is predicted and experimentally proved to result in the loss of exon 5 by improper splicing (Figure 1D).

Western blotting revealed that expression level of HADHB from the proband's fibroblast is quite lower than those from controls (Figure 1E). Additionally, Western blotting revealed that the mutant proteins expressed in HEK293 cells are more unstable than wild-type (Additional file 2: Figure S1). Cyclohexamide treatment demonstrated that the half-life of wild-type $H A D H B$ is quite short (less than $2 \mathrm{hr}$ ). Thus these results are consistent with the lowered level of $H A D H B$ in patients.

\section{Clinical manifestations}

The proband (Figure 1A, II-2) was born from nonconsanguineous Korean parents. He noticed lower leg weakness, which resulted in bilateral foot drop and gait disturbance at 5 years. At 12 years old, he used a walker due to progressively impaired gait. Neurologic examination at 34 years of age revealed that muscle weakness and atrophy began and the distal part of the lower limbs was prominently involved. Toe and heel gait defects were found. Vibration and position senses were more severely disturbed than pain and temperature senses. Deep tendon reflexes were absent in all extremities, but pathologic reflexes were not found. An echocardiogram showed normal and there was no evidence of intermittent rhabdomyolysis or retinal dysfunction. Ophthalmological examination including dilated fundus examination was done in both patients, but pigmentary retinopathy was not observed. Laboratory findings showed a total carnitine level of 33.4 $\mu \mathrm{mol} / \mathrm{L}$ (reference interval: 45-91 $\mu \mathrm{mol} / \mathrm{L}$ ), a free carnitine level of $24.5 \mu \mathrm{mol} / \mathrm{L}$ (reference interval: 36-74 $\mu \mathrm{mol} / \mathrm{L}$ ), and acylcarnitine level of $10.9 \mu \mathrm{mol} / \mathrm{L}$ (reference interval: 36-74 $\mu \mathrm{mol} / \mathrm{L}$ ).

His 37-year-old elder sister (II-1) began with distal limb weakness and gait disability from 6 years old and became to use a walker from 15 years old. At 23 years, she underwent an operation of bilateral feet due to walking difficulties. When we examined her at 37 years, she also revealed muscle weakness and atrophies of bilateral distal muscles of the upper and lower limbs. Loss of sensory and tendon reflexes were similar to the proband. The other family members were proven normal by careful clinical and electrophysiological examination.

The nerve conduction studies (NCSs) revealed that motor nerve conduction velocities (MNCVs) of median, ulnar, peroneal and tibial nerves in both patients were abnormal (Table 1). Except median nerve of II-1, all compound muscle action potentials (CMAPs) were below the normal range. Sensory nerve action potentials (SNAPs) of all tested nerves were lost. Needle electromyography (EMG) was compatible with neuropathy with fibrillation potentials and neurogenic motor unit action potentials (MUAPs).

Table 1 Electrophysiological features of patients with mutations in HADHB gene

\begin{tabular}{|c|c|c|c|c|}
\hline Patients & $\mathrm{II}-1$ & II-2 & & Normal value \\
\hline Age at exam (years) & 37 & 32 & 34 & \\
\hline \multicolumn{5}{|l|}{ Median nerve } \\
\hline TL (ms) & 4.2 & 4.1 & 4.1 & $<3.9$ \\
\hline CMAP (mV) & 7.2 & 2.2 & 3.6 & $>6.0$ \\
\hline $\mathrm{MNCV}(\mathrm{m} / \mathrm{s})$ & 44.9 & 40.6 & 41.9 & $>50.5$ \\
\hline F-wave (ms) & 36.8 & 55.0 & 38.8 & $<28.0$ \\
\hline \multicolumn{5}{|l|}{ Ulnar nerve } \\
\hline TL (ms) & 3.5 & 4.0 & 4.0 & $<3.0$ \\
\hline CMAP (mV) & 5.7 & 5.2 & 5.8 & $>8.0$ \\
\hline MNCV (m/s) & 40.4 & 43.1 & 43.9 & $>51.1$ \\
\hline F-wave (ms) & 33.2 & 36.8 & 34.4 & $<29.0$ \\
\hline \multicolumn{5}{|l|}{ Peroneal nerve } \\
\hline TL (ms) & A & A & A & $<5.3$ \\
\hline CMAP (mV) & A & A & A & $>1.6$ \\
\hline MNCV (m/s) & A & A & A & $>41.2$ \\
\hline F-wave (ms) & A & A & A & $<49.0$ \\
\hline \multicolumn{5}{|l|}{ Tibial nerve } \\
\hline TL (ms) & 3.9 & A & A & $<5.4$ \\
\hline CMAP $(\mathrm{mV})$ & 0.2 & A & A & $>6.0$ \\
\hline $\mathrm{MNCV}(\mathrm{m} / \mathrm{s})$ & 34.0 & A & A & $>41.1$ \\
\hline F-wave (ms) & 63.0 & A & A & $<52.1$ \\
\hline \multicolumn{5}{|l|}{ Median sensory nerve } \\
\hline $\operatorname{SNAP}(\mu \mathrm{V})$ & A & A & A & $>8.8$ \\
\hline $\mathrm{SNCV}(\mathrm{m} / \mathrm{s})$ & A & A & A & $>39.3$ \\
\hline \multicolumn{5}{|l|}{ Ulnar sensory nerve } \\
\hline SNAP $(\mu V)$ & A & A & A & $>7.9$ \\
\hline $\mathrm{SNCV}(\mathrm{m} / \mathrm{s})$ & A & A & A & $>37.5$ \\
\hline \multicolumn{5}{|l|}{ Sural nerve } \\
\hline $\operatorname{SNAP}(\mu \mathrm{V})$ & A & A & A & $>6.0$ \\
\hline $\mathrm{SNCV}(\mathrm{m} / \mathrm{s})$ & A & A & A & $>32.1$ \\
\hline
\end{tabular}

Bold character indicates abnormal values. $A$, absent potentials; $T L$, terminal latency; CMAP, compound muscle action potential; MNCV, motor nerve conduction velocity; SNAP, sensory nerve action potential; SNCV, sensory nerve conduction velocity; NP, no potential. 


\section{Histopathological findings}

Histopathological analysis of the distal sural nerve was performed in the proband at 34 years of age. Semi-thin transverse sections with toluidine blue stain showed absence of large myelinated fibers (MFs) with remaining medium and small-sized MFs, and occasionally noted regenerating axonal clusters (Figure 2A). Thin MFs were frequently noted and remaining MFs $\left(4,366 / \mathrm{mm}^{2}\right)$ were less than control (45-year-old male: $7,300 / \mathrm{mm}^{2}$ ). The average of diameter of MFs and MF\% area were also quite lower than control (Figure 2B). Electron microscopic examination revealed MFs with pseudo-onion bulb formation, occasionally noted regenerating clusters, and rarely noted demyelinated axon and thin MFs (Figures 2C and D). Several myelinated and unmyelinated axons showed swelling or vacuolization of axoplasm, swollen or abnormal mitochondria, and abnormal membranous structures.

\section{Lower limb MRI findings}

Brain and pelvic MRIs showed normal findings (data not shown), but lower limb MRIs of both patients revealed hyperintense signal abnormalities (Figure 3). T1-weighted images demonstrated selective muscle atrophies with signal changes, which were more prominent in lower leg muscles than thigh or pelvic muscles, thereby being consistent with the hypothesis of length-dependent axonal neuropathy. At the thigh level, there were severe involvements of the semitendinosus, sartorius and gracilis muscles, but a sparing of vastus group and adductor muscles. The lower leg MRI revealed marked involvement of tibialis anterior, peronei, extensor digitorum and halluces longus muscles; however, soleus muscles were sparing.

\section{Discussion}

Mitochondrial trifunctional protein (MTP) exerts a significant catalytic activity for last three steps of long chain
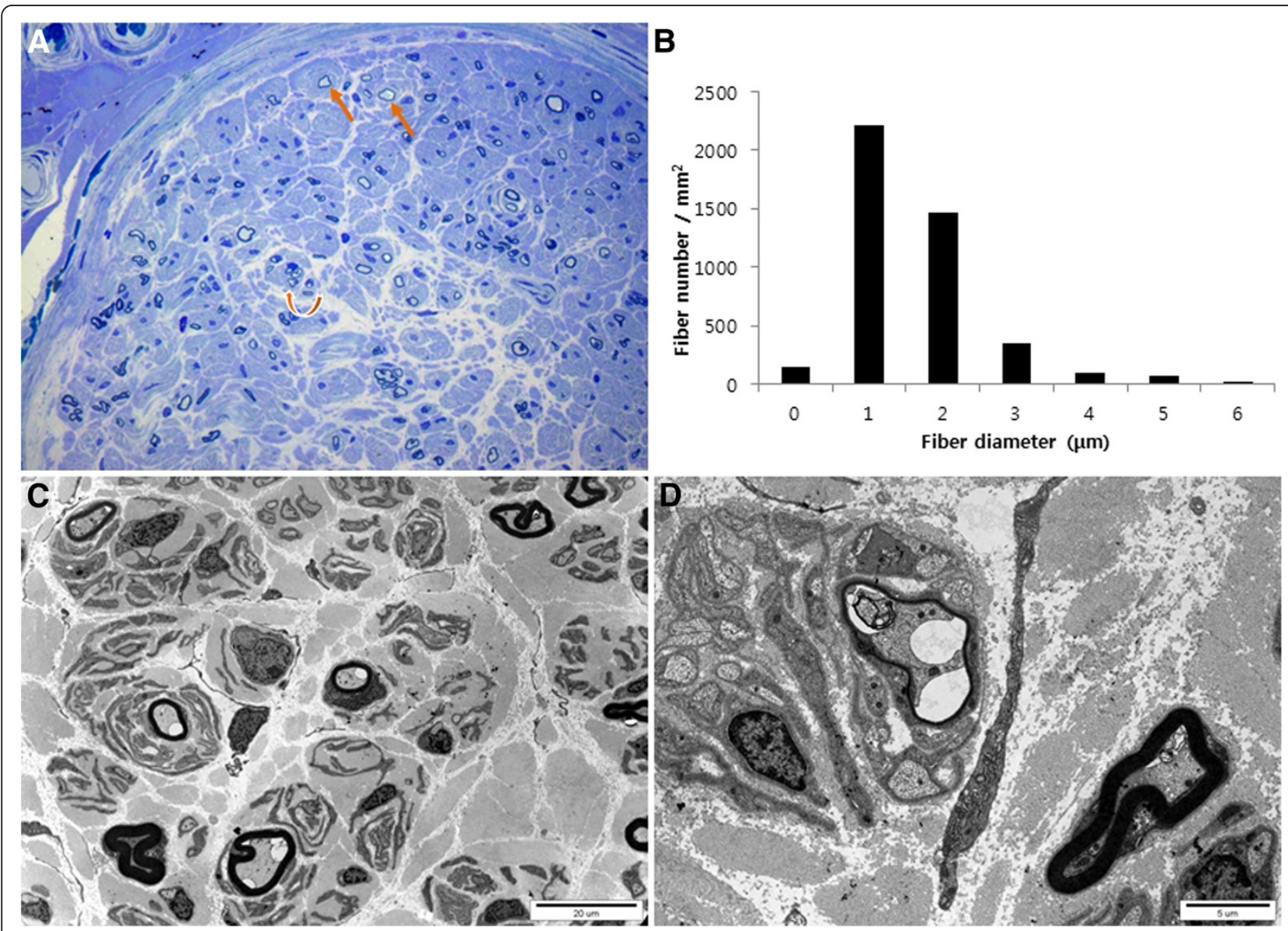

Figure 2 Histopathological findings of distal sural nerve biopsy in II-2. (A) Toluidine blue-stained semi-thin transverse section revealed absence of large MFs and remaining medium and small-sized MFs with frequently noted thin MFs (arrows) and occasionally noted axonal clusters (curved arrow). (B) Histogram showed unimodal distribution pattern of MF diameter. (C and D) Electron micrographs revealed MFs with pseudo-onion bulb formation and a thin MF compatible with remyelination. Original magnifications: A, $\times 400 ; \mathbf{C}, \times 3000$; and $\mathbf{D}, \times 10000$ 

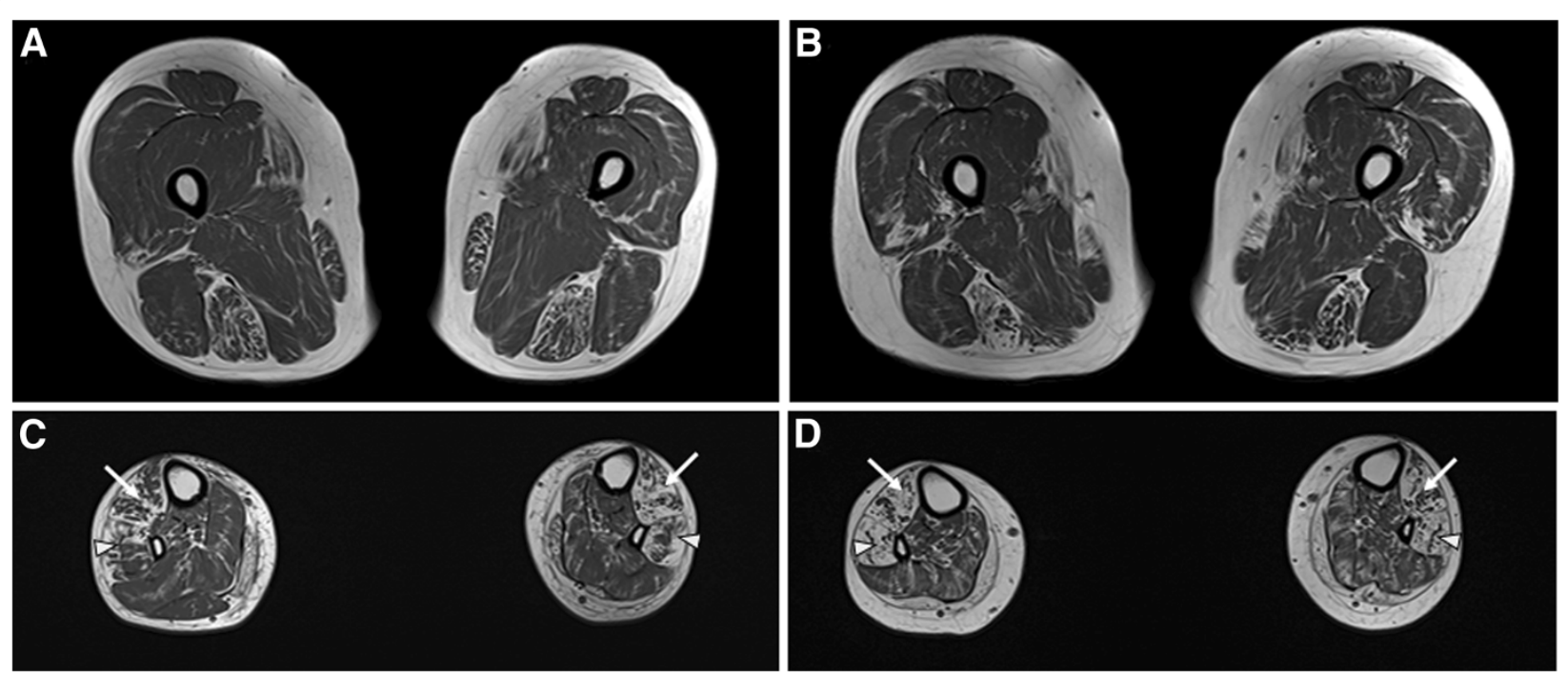

Figure 3 Thigh and lower leg MRIs in II-1 (A and C) and II-2 (B and D). (A and B) At the thigh level, MRls demonstrated severe muscle atrophied with signal changes of semitendinous muscles of posterior compartment, gracilis muscle of medial compartment, and sartorius muscle of anterior compartment. (C and D) The lower leg MRIs revealed selective severe muscle atrophy with signal change of anterior (arrow; tibialis anterior, extensor digitorum and halluces longus muscles) and lateral (arrowhead; peronei muscles) compartments. However, posterior compartment muscles (soleus and tibialis posterior muscles) were sparing in the later stages.

acyl-CoA fatty acids and consists of heterooctamer of $\alpha$-subunit $(H A D H A)$ and $\beta$-subunit $(H A D H B)$ [10]. To date, 33 mutation sites in $H A D H B$ gene have been reported [17] and these mutation-associated phenotypes exhibit clinical heterogeneity, which can be mainly subdivided into three distinctive groups: severe neonatal presentation with cardiomyopathy, hepatic form with recurrent hypoketotic hypoglycemia, and later-onset axonal sensory neuropathy with episodic myoglobinuria [11].

Several research groups have attempted to elucidate the underlying genotype-phenotype correlation of the $H A D H B$ gene. Spiekerkoetter et al. analyzed mutations from 15 patients and linked clinical phenotype with the location of the mutation [18]. They postulated that mutations in the outer loop were present only in milder forms. In addition, they suggested that the degree of reduction of cross-reactive immunological material (CRIM) is also correlated with the severity of clinical presentation. Purevsuren et al. also demonstrated the positive correlations between residual enzymatic activity and phenotypic severity using in vitro functional study [19]. Collectively, although MTP deficiency is recessive and highly heterogeneous, there are strong correlations between phenotypic severity and residual enzymatic activity or CRIM amounts of mutant proteins.

According to the crystal structure of HADHB, present mutation, Arg229Leu, locates in the outer loop [20]. This implicates that Arg229Leu mutation might mildly affect LCKT activity, while splicing variant of C.210-1G > C, which results in deletion of 15 amino acids, was predicted to have severely affected LCKT activity. In contrast to these predictions, however, overexpression of these mutants revealed that mutant proteins primarily possess lower stability than wild type. These results might come from the conformation changes of their structures, thereby translated misfolded proteins are subsequently degraded by proteases. Therefore, the loss of the enzymatic activity by the mutations might be greater than predicted.

To unveil the effect of $H A D H B$ deficiency on peripheral nerve, we first analyzed the expression of $H A D H B$ in distal sural nerve. Transcriptome analysis from 14 control samples confirmed the expression of $H A D H B$ in the distal sural nerve (data not shown). In addition, we tried to demonstrate the effect of $H A D H B$ deficiency on peripheral nerve; however, the effects of $H A D H B$ knockdown on the responses to oxidative stress and cell proliferation of mouse motor neuron were not within statistically significant ranges (data not shown). Therefore, further analyses are needed to elucidate the pathophysiological mechanism of $H A D H B$ mutation-mediated peripheral neuropathy.

Clinical and histopathological features of the present patients were consistent with an early-onset axonal sensorimotor CMT neuropathy. Both patients revealed the age at onset below 10 years old, and showed typical clinical phenotypes of CMT including predominant distal muscle weakness and atrophy, pes cavus, and steppage gait (Additional file 3: Figure S2). Nerve conduction studies in both of them were compatible with motor and sensory neuropathies. However, they did not have any other previously reported symptoms of $H A D H B$ patients, such as a rhabdomyolysis, cardiomyopathy, hypoketotic 
hypoglycemia, metabolic encephalopathy, liver dysfunction, nor pigmentary retinopathy [11]. Moreover, distal sural nerve biopsy in II-2 patient revealed typical axonal neuropathy. It is noteworthy that swollen or abnormal mitochondria were noted in several MFs and unmyelinated axons, of which significance was uncertain. Previously loss of MFs with features of Wallerian degeneration [21] and axonal and myelin degeneration [18] were reported in $H A D H B$ patients. MRI analysis indicated a muscular involvement in the present patients with $H A D H B$ mutation. In addition, lower limb MRI findings revealed severe muscle atrophy with hyperintense signal changes in the lower leg muscles than those in the thigh or hip muscles, which was compatible with the typical features of the length-dependent axonal degeneration.

\section{Conclusion}

In conclusion, we first report that a heterozygous mutation in $H A D H B$ causes early-onset axonal CMT without any typical clinical symptom of MTP based on the data from WES, MRI, and histopathological and electrophysiological analysis. Thus our findings suggest that $H A D H B$ gene can be also filed as a causative of CMT, which expands the clinical spectrum of both $H A D H B$ related disease and hereditary motor and sensory neuropathy.

\section{Additional files}

Additional file 1: Table S1. Summary of exome sequencing data for 5 samples. Table S2. Polymorphic functionally significant variants in CMT-relevant genes.

Additional file 2: Figure S1. (A) Western blotting of wild-type (WT), c.686G > T and c.210-1G > C mutants expressed in HEK293 cells. Cyclohexamide (CHX) was treated for indicated time. First lane, cells transfected with pCMV-myc control vector. (B) Western blotting of proband's fibroblast after treatment of CHX. Ctr5-7, fibroblast from CMT patients with mutation in other than HADHB gene.

Additional file 3: Figure S2. Leg pictures of the patient (II-2). The patient showed prominent distal muscle atrophies of both lower extremities and revealed typical CMT phenotypes of pes cavus, and steppage gait.

\section{Competing interests}

The authors declare that they have no competing interests.

\section{Authors' contributions}

Study concept and design: CKW, and CB-O. Acquisition of data: $\mathrm{HYB}$, LUH, PJ-M, CY-R, HYS, YBR, YJH, and KH. Analysis and interpretation of data: HYB, YJH, KH, PJ-M, CS-C, CKW, and CB-O. Drafting of the manuscript: HYB, CKW, and CB-O. Critical revision of the manuscript for important intellectual content: JS-C. Obtained funding: CKW, and CB-O. Administrative, technical, and material support: LJH, CY-R, HYS, YBR, and PJ-M. Study supervision: CKW and CB-O. All authors read and approved the final manuscript.

\section{Acknowledgements}

This study was supported by the Korean Health Technology R\&D Project, Ministry of Health \& Welfare, Republic of Korea (A120182).

\section{Author details}

'Department of Neurology, Samsung Medical Center, Sungkyunkwan University School of Medicine, 50 Ilwon-dong Gangnam-Gu, Seoul 135-710,
Korea. ${ }^{2}$ Department of Biological Science, Kongju National University, 182 Sinkwan-dong, Gongju 314-701, Korea. ${ }^{3}$ Department of Neurology, School of Medicine, Ewha Womans University, Seoul, Korea. ${ }^{4}$ Department of Biochemistry, School of Medicine, Ewha Womans University, Seoul, Korea. ${ }^{5}$ Department of Radiology, School of Medicine, Ewha Womans University, Seoul, Korea. ${ }^{6}$ Department of Pathology, School of Medicine, Ewha Womans University, Seoul, Korea.

Received: 30 January 2013 Accepted: 28 November 2013 Published: 5 December 2013

\section{References}

1. Skre H: Genetic and clinical aspects of Charcot-Marie-tooth's disease. Clin Genet 1974, 6:98-118.

2. Lupski JR, Garcia CA: Charcot-Marie-Tooth peripheral neuropathies and related disorders. In The Metabolic and Molecular Bases of Inherited Disease. Volume 4. 8th edition. Edited by Scriver CR, et al. New York: Mc Graw Hill; 2000:5759-5788.

3. Patzkó A, Shy ME: Update on Charcot-Marie-tooth disease. Curr Neurol Neurosci Rep 2011, 11:78-88.

4. Züchner S, Vance JM: Mechanisms of disease: a molecular genetic update on hereditary axonal neuropathies. Nat Clin Pract Neurol 2006, 2:45-53.

5. Pareyson D, Marchesi C: Diagnosis, natural history, and management of Charcot-Marie-tooth disease. Lancet Neurol 2009, 8:654-667.

6. Pareyson D: Axonal Charcot-Marie-tooth disease: the fog is only slowly lifting. Neurology 2007, 68:1649-1650.

7. Montenegro G, Powell E, Huang J, Speziani F, Edwards YJ, Beecham G, Hulme W, Siskind C, Vance J, Shy M, Züchner S: Exome sequencing allows for rapid gene identification in a Charcot-Marie-tooth family. Ann Neurol 2011, 69:464-470.

8. Beetz C, Pieber TR, Hertel N, Schabhüttl M, Fischer C, Trajanoski S, Graf E, Keiner S, Kurth I, Wieland T, Varga RE, Timmerman V, Reilly MM, Strom TM, Auer-Grumbach M: Exome sequencing identifies a REEP1 mutation involved in distal hereditary motor neuropathy type V. Am J Hum Genet 2012, 91:139-145.

9. Choi BO, Koo SK, Park MH, Rhee H, Yang SJ, Choi KG, Jung SC, Kim HS, Hyun YS, Nakhro K, Lee HJ, Woo HM, Chung KW: Exome sequencing is an efficient tool for genetic screening of Charcot-Marie-tooth disease. Hum Mutat 2012, 33:1610-1615.

10. Kamijo T, Aoyama T, Komiyama A, Hashimoto T: Structural analysis of CDNAs for subunits of human mitochondrial fatty acid beta-oxidation trifunctional protein. Biochem Biophys Res Commun 1994, 199:818-825.

11. Dionisi-Vici C, Garavaglia B, Burlina AB, Bertini E, Saponara I, Sabetta G, Taroni F: Hypoparathyroidism in mitochondrial trifunctional protein deficiency. J Pediatr 1996, 129:159-162.

12. den Boer ME, Dionisi-Vici C, Chakrapani A, van Thuijl AO, Wanders RJ, Wijburg FA: Mitochondrial trifunctional protein deficiency: a severe fatty acid oxidation disorder with cardiac and neurologic involvement. J Pediatr 2003, 142:684-689

13. Schwab KO, Ensenauer R, Matern D, Uyanik G, Schnieders B, Wanders RA Lehnert W: Complete deficiency of mitochondrial trifunctional protein due to a novel mutation within the beta-subunit of the mitochondrial trifunctional protein gene leads to failure of long-chain fatty acid beta-oxidation with fatal outcome. Eur J Pediatr 2003, 162:90-95.

14. Birouk N, LeGuern E, Maisonobe T, Rouger H, Gouider R, Tardieu S, Gugenheim M, Routon MC, Léger JM, Agid Y, Brice A, Bouche P: X-linked Charcot-Marie-tooth disease with connexin 32 mutations: clinical and electrophysiologic study. Neurology 1998, 50:1074-1082.

15. Shy ME, Blake J, Krajewski K, Fuerst DR, Laura M, Hahn AF, Li J, Lewis RA, Reilly M: Reliability and validity of the CMT neuropathy score as a measure of disability. Neurology 2005, 64:1209-1214.

16. Chung KW, Hyun YS, Lee HJ, Jung HK, Koo H, Yoo JH, Kim SB, Park Cl, Kim HN, Choi BO: Two recessive intermediate Charcot-Marie-tooth patients with GDAP1 mutations. J Peripher Nerv Syst 2011, 16:143-146.

17. Boutron A, Acquaviva C, Vianey-Saban C, de Lonlay P, de Baulny HO, Guffon N, Dobbelaere D, Feillet F, Labarthe F, Lamireau D, Cano A, de Villemeur TB, Munnich A, Saudubray JM, Rabier D, Rigal O, Brivet M: Comprehensive cDNA study and quantitative analysis of mutant HADHA and HADHB transcripts in a French cohort of 52 patients with mitochondrial trifunctional protein deficiency. Mol Genet Metab 2011, 103:341-348. 
18. Spiekerkoetter U, Sun B, Khuchua Z, Bennett MJ, Strauss AW: Molecular and phenotypic heterogeneity in mitochondrial trifunctional protein deficiency due to beta-subunit mutations. Hum Mutat 2003, 21:598-607.

19. Purevsuren J, Fukao T, Hasegawa Y, Kobayashi H, Li H, Mushimoto Y, Fukuda S, Yamaguchi S: Clinical and molecular aspects of Japanese patients with mitochondrial trifunctional protein deficiency. Mol Genet Metab 2009, 98:372-377.

20. Mathieu M, Modis Y, Zeelen JP, Engel CK, Abagyan RA, Ahlberg A, Rasmussen B, Lamzin VS, Kunau WH, Wierenga RK: The 1.8A crystal structure of the dimericperoxisomal 3-ketoacyl-CoA thiolase of Saccharomyces cerevisiae: implications for substrate binding and reaction mechanism. J Mol Biol 1997, 273:714-728.

21. Bertini E, Dionisi-Vici C, Garavaglia B, Burlina AB, Sabatelli M, Rimoldi M, Bartuli A, Sabetta G, DiDonato S: Peripheral sensory-motor polyneuropathy, pigmentary retinopathy, and fatal cardiomyopathy in long-chain 3-hydroxy-acyl-CoA dehydrogenase deficiency. Eur J Pediatr 2010, 151:121-126.

doi:10.1186/1471-2350-14-125

Cite this article as: Hong et al:: A compound heterozygous mutation in HADHB gene causes an axonal Charcot-Marie-tooth disease. BMC

Medical Genetics 2013 14:125.

\section{Submit your next manuscript to BioMed Central and take full advantage of:}

- Convenient online submission

- Thorough peer review

- No space constraints or color figure charges

- Immediate publication on acceptance

- Inclusion in PubMed, CAS, Scopus and Google Scholar

- Research which is freely available for redistribution 